\title{
El Narcotal en la Práctica Obstétrica
}

\author{
Hospital de San José de Bogotá - Comunicación Preliminar \\ 19961 \\ Dr. Miguei A. Fernández-Bastidas
}

La primera década de la anestesia obstétrica se remonta a ciento veinte años atrás con las publicaciones que hiciera Morton, Wells, Crawford Simpson, Snow y Oliver Wendell Holmes, quienes con escasos recursos técnicos utilizaron el éter, el cloroformo, el óxido nitroso y el cloruro de etilo.

Entre nosotros hasta hace relativamente pocos años, al igual que en el resto del mundo, a la anestesia obstétrica no se le daba suficiente importancia y su administración se confiaba a personal con poca experiencia y sin preparación especializada; solamente y desde hace pocos años tenemos funcionando en nuestro Servicio un selecto grupo de anestesiólogos que haciendo parte del Equipo Médico de turno y con su inestimable colaboración, hemos logrado controlar los posibles casos de neumonitis por aspiración y demás causas de morbilidad y mortalidad materna achacables a una pobre técnica anestésica.

En nuestro país gracias al aumento creciente en el número de centros de asistencia cibstétrica en los últimos diez años, en especial de aquellos dedicados a la atención de pacientes semiprivadas (clientela de seguros sociales), el avance de las técnicas anestésicas en obstetricia ha sido notable. (1)

La anestesia raquídea tan aconsejada por Hingson (EE. UU.), y cuyo auge en Colombia ha sido notable aún desde antes del curso especializado de anestesia dictado en Bogotá por este destacado investigador, ha dado pleno respaldo a las actuales favorables estadísticas nacionales.

En el momento actual con los interesantes estudios llevados a cabo en Cleveland por Hendricks y Kretchner utilizando los métodos inscriptores de los uruguayos Alvarez y Caldeiro, se han obtenido gráficas de la contracción uterina durante la inducción anes- 
tésica (2) aclarando una vez por todas no pocos conceptos erróneos relacionados con su farmacodinamia.

Hingson y col. (3), estudiando igualmente la cantidad de ciclopropano asociado a barbitúricos endovenosos titulados a nivel de la llamada "barrera placentaria", han demostrado que su paso a través de ella, justifica la importancia dada por Quillingen a la oxigenoterapia que al obtener el paso a la sangre fetal del oxígeno inhalado por la madre durante la asociación con el anestésico de base, hace más atenuados y controlables los efectos anoxémicos de la droga utilizada. Iguales experiencias son las publicadas por Lipsitz de Sur Africa (5) quien utiliza en forma sistemática la oxigenoterapia en la resucitación fetal de casos en que la madre ha recibido anestesia general, obteniendo análogos resultados.

Como se puede ver, éstos y otros numerosos estudios que no es del caso enumerar, han contribuído a disminuír cada vez más el índice de mortalidad fetal por anestesia, que en algunas estadísticas extranjeras llegaban hasta el $10 \%$ al $20 \%$ de nacidos vivos y durante su primer año de vida.

Hoy pues, está plenamente demostrado (Henricus y Stander) (6), que todo anestésico es potencialmente peligroso para el feto, al pasar la "barrera placentaria" y producir fallas en su oxigenación, además de caídas bruscas tensionales maternas que en caso de llegar a ser inferiores a los $8 \mathrm{~mm}$. de mercurio comprometerán la vida del hijo.

Dicho lo anterior, cabe preguntar; cuáles son las condiciones ideales para una segura y efectiva anestesia obstétrica? La respuesta la podremos obtener transcribiendo las condiciones dadas por Hingson para una segura aplicación. (7)". I - La constitución de un Equipo formado por obstetras, anestesiólogos y enfermeras entrenadas con turnos de veinticuatro horas. II - La dotación de equipo y material necesario para todas las medidas profilácticas y de terapéutica restaurativa que puedan dominar crisis respiratorias o vasomotoras tanto maternas como del recién nacido en forma instantánea. Tales como la pronta oxigenación del $100 \%$ lograda en la cabecera de la paciente en trabajo y todas las facilidades de aspiración de líquidos o de vómito. Con la indispensable condición de que el grupo médico sepa en todo momento aplicar tales recursos. III - La necesidad de dar instrucción a las pacientes sobre la importancia del apropiado cuidado 
prenatal aclarando por medio de explicaciones el mecanismo del parto y del alumbramiento normales, creando en tal forma una útil tranquilidad mental de la embarazada que contribuirá grandemente a una mayor cooperación que evitará la integración de alimentos inmediatamente antes del trabajo activo del parto, constituyéndose así la paciente como un cuarto miembro del Equipo Obstétrico. Siendo necesaria la instrucción en todos los casos que sea factible del marido, quien como compañero de su esposa durante el parto, deberá ayudar a poner en práctica tales indicaciones. IV - El Equipo Médico a cuyo cuidado está la moderna paciente obstétrica deberá estar preparado para poner en práctica una de las siguientes conductas: a) Anestesia general instantánea y segura a fin de eliminar el "dolor" fisiológico obstétrico de máxima intensidad. b) Bloqueo raquídeo instantáneo o conducción anestésica local, y c) Técnicas sicológicas de apoyo para la paciente que no desee o no requiera analgesia 0 anestesia medicamentosa para el parto".

Como puede verse por lo anterior, las cuatro condiciones enumeradas son las ideales para ser aplicadas en todo Servicio Obstétrico bien organizado y son las que han sido puestas en consideración en el Hospital de San José. Las dos primeras tienen en el momento presente vigencia; la relativa a la parte sicológica podemos asegurar que de acuerdo con nuestras publicaciones (8), (9), fuimos los primeros en Colombia en ponerlas en práctica en clientela hospitalaria. y si en la actualidad no se tienen como rutina, no es por dejar de reconocerles la importancia que merecen de todo punto de vista.

Siendo el principal objeto del presente trabajo la presentación de resultados, fruto de nuestra experiencia lograda con la anestesia endovenosa por medio del Iso propil B bromatil N. metilmalonilcarbaminato de sodio (Narcotal) en nuestro Servicio de Obstetricia y Ginecología, haremos un resumen breve de este tipo de anestesia.

Desde la primera narcosis por vía endovenosa llevada a cabo por Oré en 1872 utilizando cloral y el uso primero de barbitúricos por Fischer y von Mering en 1903, se han sintetizado mediante el cambio de radicales, varios miles de barbitúricos distintos, de entre los cuales unos cincuenta con marcada acción narcótica. Clínicamente se diferencian unos de otros sobre todo por 
su duración que a su vez depende de la distinta velocidad con que se efectúa el desdoblamiento y la eliminación. Sin embargo, hasta el descubrimiento de los barbitúricos de acción ultracorta, la narcosis intravenosa no alcanzó un puesto central dentro de la técnica moderna de la narcosis.

El pentobarbital, de muy común utilización desde hace ya largos años en la práctica obstétrica, después de las publicaciones de Mc Allister y Flowers quienes analizaron 7.793 casos sin encontrar contraindicaciones absolutas maternas ni fetales, ha dado lugar a que Eastman (10) declare que: "el pentobarbital posee muchas ventajas sobre otra forma de anestesia" alegrándole ver "reafirmados tales méritos de seguridad para el parto". Igualmente Whyte del Canadá (11) y Romero y col. de Venezuela (12) en series que comprendían en conjunto más de dos millares de casos, acreditan su uso con ligeras excepciones.

El Narcotal, droga de más reciente síntesis es un barbitúrico análogo pero de acción ultracorta. Administrado por vía endovenosa produce una narcosis de efecto rápido que se puede controlar por medio de la velocidad de la inyección y que proporciona un despertar rápido una vez terminada la inyección. Narcotal produce en la gran mayoría de los casos como lo veremos al analizar los casos presentados, un adormecimiento tranquilo y agradable sin estados de excitación ni vómitos molestos. La respiración es tranquila y no se origina tampoco salivación molesta aún en los casos no premedicados con atropina. El método de aplicación es sencillo y desde luego no hay peligro de explosión. (13).

\section{MATERIAL Y METODO}

Los primeros casos seleccionados, dadas las características del anestésico de rápida inducción y acción ultracorta, fueron aquellos en que una rápida intervención exigía tal característica anestésica. Por tal motivo las series presentadas en esta comunicación preliminar solamente comprenden casos de aborto incompleto, en los cuales la fuerte hemorragia y en la mayoría el estado precario de las pacientes imponía una acción inmediata.

A pesar de un número hasta el momento muy limitado, por tratarse de solamente catorce casos analizados, presentamos sin comentario los datos obtenidos. 
El método seguido en todos los casos fué la aplicación de la droga sin diluír y directamente en el torrente venoso. Los planos de anestesia logrados no fueron en ningún caso hasta límites tóxicos y el tercer plano de narcosis con pérdida del reflejo visual, apenas se encontró en un número reducido de casos.

Vale la pena aclarar que todos los fármacos de este tipo, más que analgesia producen hipnosis, notándose pocas variaciones tensionales al igual que conservación del débito cardíaco normal, motivos por los cuales han sido aconsejados en casos de cardiópatas compensadas. En cambio los fenómenos depresivos respiratorios y la frecuencia de laringoespasmos son más frecuentes con su uso. (14)

Por tales motivos, en todos nuestros casos la asociación de oxigenoterapia ha sido tenida como irreemplazable, pudiendo controlar así cualquier estado de hipoxia.

\section{RESULTADOS}

Isopropil $\mathrm{B}$ bromatil $\mathrm{N}$ metilmalonilcarbaminato de sodio (Narcotal), aplicación endovenosa.

Dosis y duración de la anestesia

$\begin{aligned} 20 \text { minutos }(10 \text { c.c. y Ciclo } 02): & \text { un caso } \\ 20 \text { minutos }(10 \text { c.c y } 02): & \text { un caso } \\ 25 \text { minutos }(10 \text { c.c. y } 02): & \text { un caso } \\ 30 \text { minutos }(10 \text { c.c. y } 02): & \text { un caso } \\ 35 \text { minutos }(10 \text { c.c. Ciclo } 02): & \text { dos casos } \\ 40 \text { minutos }(15 \text { c.c. Ciclo } 02): & \text { un caso } \\ 45 \text { minutos }(5 \text { c.c. Ciclo } 02): & \text { un caso } \\ 45 \text { minutos }(15 \text { c.c. Ciclo } 02): & \text { un caso } \\ 60 \text { minutos }(10 \text { c.c. Ciclo } 02): & \text { dos casos } \\ 60 \text { minutos }(15 \text { c.c. } 02): & \text { un caso } \\ 90 \text { minutos }(15 \text { c.c. Ciclo } 02): & \text { un caso } \\ 120 \text { minutos }(15 \text { c.c. Ciclo } 02): & \text { un caso }\end{aligned}$

Resumen:

Narcotal 5 c.c. + Ciclo 02: un caso. Promedio tiempo: 45'

Narcotal 10 c.c. +02 : tres casos. Promedio tiempo: 25' 
Narcotal 15 c.c. $+02 \cdot$ un caso. Promedio tiempo: 60'

Narcotal 10 c.c. + Ciclo 02: cinco casos. Promedio tiempo: 42'

Narcotal 15 c.c. + Ciclo 02 : cuatro casos. Prom. tiempo: 73,8'

Premedicación anestésica

Escopolamina:

Demerol escopolamina:

Gardenal atropina:

Atropina :

Gardenal :

Indicación anestésica

Raspado uterino:

Profundidad anestésica

Primer plano anestésico:

Segundo plano anestésico:

Asociación anestésica

Con ciclopropano como agente secundario:

Con ciclopropano como agente terciario:

Sin asociación anestésica:

Complicaciones durante la anestesia

a) Hipotensión:

cuatro casos tratados así:

un caso: disminución del Narcotal a emplear

un caso: Sangre 500 c.c., Ergotrate vía i.v.

un caso: Sangre 500 c.c.

un caso: Sol dextrosa $5 \%$ en agua 500 c.c. y 02 .

Nota: los dos primeros casos habían recibido 15 c.c. de droga; el resto 10 c.c. - Todos los casos presentaban hemorragia vaginal abundante.

b) Paro Respiratorio:

Un caso tratado con cafeína endovenosa, intubación 02 a presión, y que había recibido 10 c.c. de droga.

c) Espasmo Laringeo:

Un caso tratado con Rubilexin 10 mgs., intubación y 02 a presión. 
Registro de Tensión Arterial

Sin modificaciones:

3 con 10 c.c. de droga

2 con 15 c.c. de droga

Ligera baja inicial:

1 con 5 c.c. de droga

2 casos con 10 c.c. de droga

2 casos con 15 c.c. de droga

Caída de más de $40 \mathrm{~mm}$ : 1 caso con 10 c.c. de droga

2 casos con 10 c.c. de droga (hemorragia)

1 caso con 15 c.c. de droga

Registro de pulso

Sin modificaciones producidas por la droga; solamente un caso achacable al estado de anemia por hemorragia.

Líquidos Suministrados

Sol. Dextrosa al $5 \%$ en agua 500 c.c.:

12 casos

Sol. Dextrosa $5 \%$ agua y Sangre 500 c.c.:

2 casos

Sol. Dextrosa 5\% agua con Veritol 500 c.c.:

1 caso

Ergotrate vía endovenosa:

9 casos

Pitocin 10 unidades vía I.V.:

1 caso

Condiciones de la paciente a la salida de la sala

Regular estado:

cinco casos

Buen estado:

nueve casos

\section{RESUMEN}

Se presenta como una comunicación preliminar un estudio llevado a cabo en el Departamento de Obstetricia y Ginecología del Hospital de San José, con pacientes intervenidas por aborto incompleto.

Se hace una revisión somera sobre las últimas experiencias publicadas en otros centros de estudio sobre la anestesia en Obstetricia, resaltando la importancia del control de la anoxia por constituír peligro inminente tanto a la madre como al feto.

Se comentan las condiciones óptimas para obtener una anestesia segura en obstetricia, recalcando sobre la preparación sicológica de la embarazada. 
Catorce casos son resumidos teniendo en cuenta la duración, dosis, profundidad anestésica, complicaciones y resultados obtenidos con la aplicación de un nuevo barbitúrico para vía endovenosa de acción ultracorta y cuya fórmula es: Isopropil B Bromatil N Metilmalonil carbaminato de sodio, conocido en el comercio como Narcotal.

\section{BIBLIOGRAFIA}

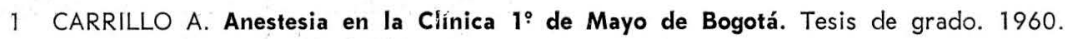

2 VASICKA A. KRETCHMER H. F. "Uterine Dynamic". Clin. Obst. and Gynec. 4: 17, 1961. Baltimore.

3 HINGSON R. A. VASICKA A. "Conduction Anesthesia and Analgesia for Obstetrics". Clin. Obst. and Gynec. 4: 87, 1961.

4 QUILLIGAN E. J. "Effects of inaternal anesthesia on fetal oxigen". Clin. Obst. and Gynec. 4: 47, 1961 Baltimore.

5 LIPSITZ P. J. "Resuscitation of the newborn Infant". Obst. and Gynec. Clin. 4: 117, 1961 Baltimore.

6 STANDER H. J. citado por HINGSON. Clin. Obst. and Gynec. 4: 14. 1961.

7 HINGSON R. A. "Foreword". Ciin. Obst. and Gynec. 4: 15, 1961.

8 CALDERON BELISARIO. "Método sicoprofiláctico en clientela hospitalaria. Primera comunicación del Hospital de San José". Rev. Col. de Obst. y Gynec. 8: 341 1957. Bogotá.

9 FERNANDEZ-BASTIDAS M. A.: "Analgesia Obstétrica por métodos sicofísicos". Hospital de San José. Obst. y Ginec. Latino-Americanas 17: 360. 1960. Buenos Aires.

10 EASTMAN N. J. Obst. y Gynec. Survey 12: 3251957.

11 WHYTE J. C. Am. J. Obst. an' Gynec. 63: 163. 1952.

12 ROMERO A. GRATERON L. B. CONTRAMAESTRE. Rev. Obst. y Ginec. Venezuela. 19: 651959.

13 ASTRA Sudertalje. Suecia. Literatura sobre Narcotal.

14 BONICA J. J Clin Obst. and Gynec. 4: 682. 1961. Baltimore. 